\title{
FIR Filter Design for Frequency Invariant Beamformers
}

\author{
Darren B. Ward, Rodney A. Kennedy, and Robert C. Williamson
}

\begin{abstract}
Two methods of implementing FIR filters for a frequency invariant beamformer are presented. Each of these methods uses a single underlying set of filter coefficients obtained directly from the desired beamformer response. One method uses multirate processing, and the other is based on a single sampling rate.
\end{abstract}

\section{INTRODUCTION}

A FREQUENCY invariant beamformer (FIB) is a beamformer in which the response is (approximately) constant over an arbitrarily wide design bandwidth. This type of beamformer is particularly useful in speech acquisition with microphone arrays, and several methods of design have been proposed [1]-[3]. In [4], a new analog technique based on approximating an ideal continuous aperture was presented. Based on that theory, two methods of designing FIR filters for use in an FIB are proposed in this letter. The first method involves multirate sampling, and the second is based on a single sampling rate.

\section{FREQUENCY INVARIANT BEAMFORMING}

The method developed in [4] is briefly outlined here for the specific case of a linear aperture. The response of a linear continuous aperture to planar waves from an angle $\theta$ measured relative to broadside is

$$
\tau(\theta, f)=\int_{0}^{x_{\max }} e^{j 2 \pi f x c^{-1} \sin \theta} \rho(x, f) d x
$$

where $\rho(x, f)$ is the aperture illumination, considered to be a continuous function of both location $x$ and frequency $f$, and $c$ is the speed of wave propagation. We assume that $\rho(x, f)=0$ for $x>x_{\max }$.

It is straightforward to show that the response of a broadband aperture remains constant if the aperture illumination in (1) is given by $\rho(x, f)=f G(x f)$, where $G(\cdot)$ is an arbitrary absolutely integrable function. Therefore, the filtering of an FIB can be broken into two parts: 1) the primary filter response, $H_{x}(f)=G(x f)$, and 2) the secondary filter response, $f$, which is independent of position.

Manuscript received March 29, 1995. This work was supported by the Australian Research Council. The associate editor coordinating the review of this letter and approving it for publication was Dr. J. M. F. Moura.

D. B. Ward and R. C. Williamson are with the Department of Engineering, Faculty of Engineering and Information Technology, Australian National University, Canberra, Australia.

R. A. Kennedy is with the Telecommunications Engineering Group, Research School of Information Sciences and Engineering, Australian National University, Canberra, Australia.

Publisher Item Identifier S 1070-9908(96)01159-5.

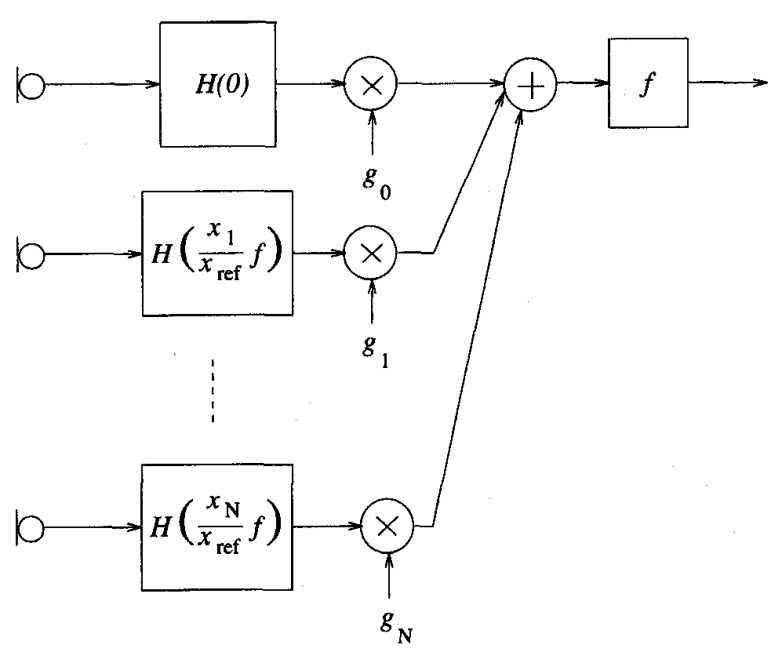

Fig. 1. Block diagram of a general linear FIB.

The most important feature of the FI aperture is that all primary filters are related by dilation, i.e., if $H_{x}(f)$ is the primary filter response at an arbitrary point on the aperture, then the primary filter response at a point $\gamma x, \gamma>0$, is given by $H_{\gamma x}(f)=G(\gamma x f)=H_{x}(\gamma f)$.

In order to design a practical FIB, it is necessary to approximate the response of the continuous aperture by providing a numerical approximation to the family of integrals described by (1). Let $\left\{x_{n}\right\}_{n=0}^{N}$ denote a set of $N+1$ sensor locations with the zeroth sensor located at $x_{0}=0$. With frequency limited to the range $\left[f_{L}, f_{U}\right]$, we can consider the following simple class of approximations:

$$
\hat{r}(\theta)=f \sum_{n=0}^{N} g_{n} H_{n}(f) e^{j 2 \pi f x_{n} c^{-1} \sin \theta}, \quad f \in\left[f_{L}, f_{U}\right]
$$

where $\hat{r}(\theta)$ is the (approximately) frequency invariant response, $g_{n}$ is a spatial weighting term to compensate for the possibly nonuniform sensor spacings, and $H_{n}(f)=G\left(x_{n} f\right)$ is the primary filter response of the $n$th sensor. The method is similar to that developed in [3]. In [4], it was shown how to obtain $g_{n}$ for the case corresponding to the trapezoidal integration method; no further discussion of $g_{n}$ will be given here.

With the FIB response thus defined, we are led to the block diagram shown in Fig. 1, in which $H(f)$ is the primary filter response at some reference location $x_{\text {ref }}$. Note that the filter response of the zeroth sensor is constant. 
The set of sensor locations can be determined by minimizing the number of sensors required while avoiding spatial aliasing [3]. In the case of a linear aperture, the sensor locations are given by

$$
x_{n}= \begin{cases}\frac{\lambda_{U}}{2} n, & 0 \leq n \leq P \\ P \frac{\lambda_{U}}{2}\left(\frac{P}{P-1}\right)^{n-P}, & P<n \leq N\end{cases}
$$

where $P \in \mathbb{N}$ is the aperture length measured in half wavelengths, $\lambda_{U}$ is the wavelength corresponding to the upper frequency of operation, and $N=P+\left[\log \left(\frac{f_{U}}{f_{L}}\right) / \log \left(\frac{P}{P-1}\right)\right]$.

\section{DESIGN OF PRIMARY FILTERS}

The most important property of the primary filters of an FIB is frequency dilation, which means that all primary filters are derived from a single reference frequency response, and hence all primary filter coefficients may be derived from a single set of coefficients.

\section{A. Multirate Method}

Let $h_{\text {ref }}[k]$ be a set of $L$ filter coefficients which produces a desired primary filter response $H_{\text {ref }}(f)$ at a reference location $x_{\text {ref }}$ with a sampling period $T$. The primary filters will have the required dilation property if the $n$th primary filter response is given by

$$
H_{n}(f)=\sum_{k=-(L-1) / 2}^{(L-1) / 2} h_{\mathrm{ref}}[k] e^{-j 2 \pi f T_{n} k}
$$

where $T_{n}=T x_{n} / x_{\text {ref }}$ is the sampling period of the $n$th sensor.

A common method of multirate sampling is to sample every sensor at the highest rate required and then to use decimation to achieve the desired sampling rate [5]. Thus, each of the primary filters would be implemented by downsampling by $\gamma_{n}=x_{n} / x_{\text {ref }}$, applying the reference primary filter, and then upsampling by $\gamma_{n}$. Note that $\gamma_{n}$ must be an integer, requiring modification of (3) such that $x_{n} / x_{\text {ref }} \in \mathbb{N}$.

The aperture length is defined to be $P$ half-wavelengths at all frequencies within the design band. Thus, the nth primary filter is (ideally) band-limited with $H_{n}(f)=0$ for $|f|>P c /\left(2 x_{n}\right)$. Ignoring the zeroth primary filter (which has a constant response), the primary filter with the widest bandwidth is located at $x_{1}=c /\left(2 f_{U}\right)$. Hence, the effective bandwidth is $P f_{U}$, requiring a sampling rate of $f_{s}=2 P f_{U}$. The reference primary filter is located at $x_{\text {ref }}=c /\left(2 f_{U}\right)$.

\section{B. Single Rate Method}

As in the multirate case, assume there is a set of reference coefficients having a desired primary filter response at some reference location. By reconstructing the continuous-time impulse response of $h_{\text {ref }}[k]$, applying the scaling property of the Fourier transform, and resampling the scaled impulse response, the $n$th set of primary filter coefficients is given by

$$
h_{n}[m]=\frac{1}{\gamma_{n}} \sum_{k=-(L-1) / 2}^{(L-1) / 2} h_{\mathrm{ref}}[k] \operatorname{sinc}\left(\frac{m}{\gamma_{n}}-k\right), \quad \gamma_{n} \geq 1
$$
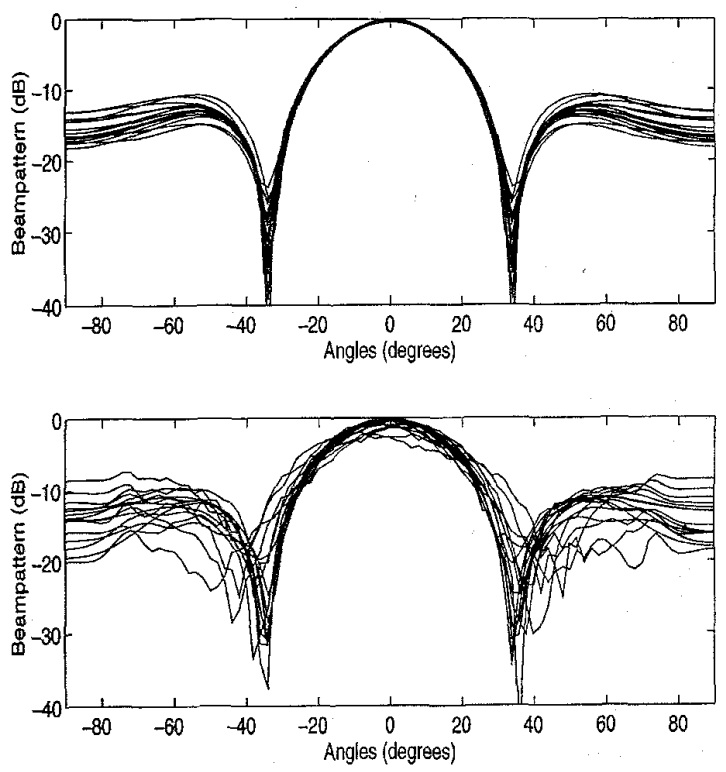

Fig. 2. Beam pattern of a 17 -element FIB at 15 frequencies within the design bandwidth: (a) Using multirate processing; (b) using a single sampling rate.

where $\gamma_{n}=x_{n} / x_{\text {ref }}$ and $\operatorname{sinc}(x)=\sin (\pi x) /(\pi x)$. For $0<\gamma_{n}<1$, the reference set of coefficients must first be convolved with the coefficients of a low-pass filter having a cutoff of $\gamma_{n} f_{s} / 2$ to avoid temporal aliasing. For $\gamma_{n}=0$, the reference coefficients are simply an impulse. The length of the $n$th primary filter should be $L \gamma_{n}$ for $\gamma_{n}>1$; a predefined number of coefficients should be used for $0<\gamma_{n}<1$ to give best results.

If the input signal is band-limited to $f_{U}$, the minimum sampling rate is $f_{s}=2 f_{U}$. Recall that (ideally) $H_{n}(f)=0$ for $|f|>P c /\left(2 x_{n}\right)$. The location of the reference sensor should be chosen such that $H_{\text {ref }}(f)=0$ for $|f|>f_{s} / 2$. Hence, for the single sampling rate FIB, the reference sensor is located at $x_{\text {ref }}=P c /\left(2 f_{U}\right)$.

\section{Determining the Coefficients of the Reference Primary Filter}

With the substitution $\rho(x, f)=f G(x f)$ and the change of variables $u=c^{-1} \sin \theta$ and $y=x f$ in (1), the Fourier transform relationship between the desired response and the aperture distribution is apparent. thus, given a desired response $r_{d}(u)$ defined in the range $U \in[-1 / c, 1 / c]$, i.e., the visible region, the required aperture distribution is $G(y)=$ $\int_{-1 / c}^{1 / c} r_{d}(u) \exp (-j 2 \pi y u) d u$. Defining $\tilde{H}_{x}(f)=G(x f)$ as the primary filter response of the sensor at $x$, it is clear that

$$
\tilde{H}_{1}(f)=\int_{-1 / c}^{1 / c} r_{d}(u) e^{-j 2 \pi f u} d u
$$

and that the impulse response of the primary filter at $x=1$ is identical to the desired FIB response, $r_{d}(\cdot)$. The set of coefficients for this primary filter can then be found by direct sampling as $h_{1}[k]=r_{d}(k T)$, where $T$ is the sampling period. By the scaling property of the Fourier transform, the coefficients of the primary filter at some location $x_{\text {ref }}$ are given 
by

$$
h_{\text {ref }}[k]=\frac{1}{x_{\text {ref }}} r_{d}\left(\frac{k T}{x_{\text {ref }}}\right), \quad x_{\text {ref }}>0 .
$$

Note that since $u \in[-1 / c, 1 / c]$, the length of the reference filter is limited.

\section{Design of SeCondary FILTer}

Because the secondary filter is a differentiator, its design is straightforward. The secondary filter should be a Type 4 FIR filter, i.e., even length with odd symmetric coefficients [6].

\section{Simulation EXAMPLES}

Two examples are presented to demonstrate the effectiveness of each of the methods. In both examples, the aperture length was $P=4$ half-wavelengths, and the design bandwidth was $200-3400 \mathrm{~Hz}$, requiring 17 sensors and a total array size of $3.4 \mathrm{~m}$ (for acoustic waves in air). A secondary filter with 12 coefficients and a uniform aperture illumination were used in both cases.

Fig. 2(a) shows the response of the multirate FIB with a maximum sampling rate of $30 \mathrm{kHz}$ and a reference filter with nine coefficients. Fig. 2(b) shows the response of the single sampling rate FIB with a sampling rate of $8 \mathrm{kHz}$. The reference filter again had nine coefficients, but each of the primary filters had a minimum of 51 coefficients (with the largest primary filter having 151 coefficients). It is seen that, in both cases, the beam pattern is relatively FI over the entire design bandwidth. The multirate method requires fewer filter coefficients but at the expense of a higher sampling rate.

\section{ACKNOWLEDGMENT}

The author thanks $\mathrm{P}$. Cheung for helping to prepare the examples.

\section{REFERENCES}

[1] J. L. Flanagan, "Beamwidth and usable bandwidth of delay-steered microphone arrays," AT\&T Tech. J., vol. 64, no. 4, pp. 983-995, Apr. 1985.

[2] M. M. Goodwin and G. W. Elko, "Constant beamwidth beamforming," in Proc. ICASSP-93, vol. 1, 1993, pp. 169-172.

[3] J. H. Doles, III and F. D. Benedict, "Broad-band array design using the asymptotic theory of unequally spaced arrays," IEEE Trans. Antennas Propagat., vol. 36, pp. 27-33, Jan. 1988.

[4] D. B. Ward, R. A. Kennedy, and R. C. Williamson, "Theory and design of broadband sensor arrays with frequency invariant far-field beam patterns," J. Acoust. Soc. Amer., vol. 97, no. 2, pp. 1023-1034, Feb. 1995 .

[5] P. P. Vaidyanathan, Multirate Systems and Filter Banks. Englewood Cliffs, NJ: Prentice-Hall, 1993.

[6] T. W. Parks and C. S. Burrus, Digital Filter Design. New York: Wiley, 1987. 